\title{
Analysis of magnetic pressure effects in atmospheres of CP stars
}

\author{
Denis Shulyak $^{1}$, G. Valyavin ${ }^{2}$, O. Kochukhov ${ }^{3}$, and T. Burlakova ${ }^{4}$ \\ ${ }^{1}$ Institute for Astronomy, Vienna University, Türkenschanzstrasse, 17, 1180 Vienna, Austria \\ email: denis@jan.astro.univie.ac.at \\ ${ }^{2}$ Korea Astronomy and Space Science Institute, 61-1, Whaam-Dong, Youseong-Gu, Taejeon, \\ Korea 305-348 \\ email: gendoz@boao.re.kr \\ ${ }^{3}$ Department of Astronomy and Space Physics, Uppsala University, Box 515, 75120 , \\ Uppsala, Sweden \\ email: Oleg.Kochukhov@fysast.uu.se \\ ${ }^{4}$ Special Astrophysical Observatory, Russian Academy of Sciences, Nizhnii Arkhyz, Karachai \\ Cherkess Republic, 369167, Russia
}

\begin{abstract}
Several dynamical processes may induce considerable Lorentz forces in atmospheres of $\mathrm{mCP}$ stars, thus modifying the hydrostatic structure of their atmospheres. This modification can be seen as characteristic rotational variability of certain spectral features such as hydrogen Balmer lines. In this work we present the first results of modeling the magnetic pressure effects in atmospheres of $\mathrm{mCP}$ stars in the framework of model atmosphere analysis with accurate treatment of microscopic properties of atmospheric plasma. We show that at least part of the rotational variability of hydrogen lines seen in high-resolution spectra of mCP stars could be attributed to the non-zero electrical currents flowing along stellar surfaces.
\end{abstract}

Keywords. Stars: chemically peculiar - stars: magnetic fields - stars: atmospheres

\section{Introduction}

Magnetic chemically peculiar $(\mathrm{mCP})$ stars display the presence of stable large-scale magnetic fields. The slow evolution of these magnetic fields (as well as other dynamical processes) may induce considerable electric currents, thus modifying the pressure balance of stellar atmospheres via the induced Lorentz forces. These forces can be detected observationally analysing the pressure sensitive spectral features like hydrogen lines (Kroll 1989). Here we present the study of hydrogen lines variability in two mCP stars $\theta$ Aur and 56 Ari within the framework of model atmospheres with magnetic pressure included.

\section{Model predictions}

To model the variation of the Balmer line profiles, we follow the approach outlined in Shulyak et al. (2007); Valyavinet al. (2004). The hydrostatic equation of stellar atmosphere in the presence of the magnetic pressure can be written as:

$$
\frac{\partial P_{\text {total }}}{\partial r}=-\rho g \pm \frac{1}{c} \lambda_{\perp} \sum_{n} c_{n} P_{n}^{1}(\mu) \sum_{n} B_{\theta}^{(n)},
$$

where $c_{n}$-the effective e.m.f. generated by the $n$-th magnetic field component at the stellar magnetic equator and $B_{\theta}$ is the horizontal field component. $\lambda_{\perp}$ is the plasma conductivity across magnetic field lines and $P_{n}^{1}(\mu)$ are the Legendre polynomials. 

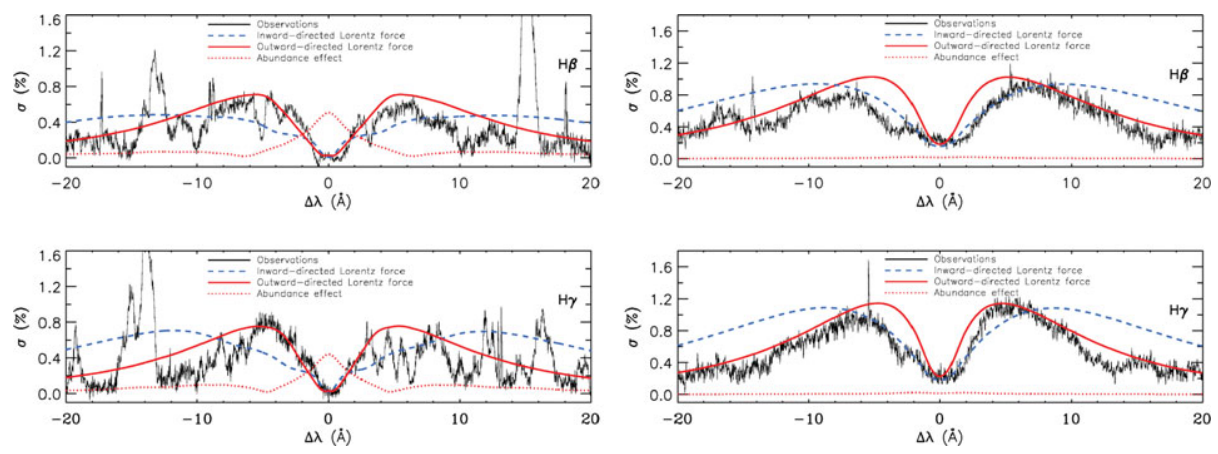

Figure 1. Standard deviation around $\mathrm{H} \beta$ and $\mathrm{H} \gamma$ lines of $\theta$ Aur (left panel) and 56 Ari (right panel) obtained under different assumptions about the direction of the Lorentz force.

Table 1. Main stellar and magnetic field parameters of $\theta$ Aur and 56 Ari. Here $i$-inclination angle, $\beta$-magnetic obliquity, $B_{\mathrm{d}}$-polar strength of the dipole component, $c_{\mathrm{q}} / c_{\mathrm{d}}$-relative strength of induced e.m.f.

\begin{tabular}{l|cccccccc}
\hline \hline & $T_{\text {eff }}$ & $\log g$ & $i^{\circ}$ & $v \sin i$ & $\beta^{\circ}$ & $B_{\mathrm{d}} \mathrm{kG}$ & $B_{\mathrm{q}} / B_{\mathrm{d}}$ & $c_{\mathrm{q}} / c_{\mathrm{d}}$ \\
\hline$\theta$ Aur & 10400 & 3.6 & 51 & 55 & 78 & 1.4 & -2 & 2.5 \\
56 Ari & 12800 & 4.0 & 70 & 160 & 82 & 1.3 & 0 & 0 \\
\hline
\end{tabular}

We applied the LLMODELS stellar model atmosphere code (Shulyak et al. 2004) to compute $\lambda_{\perp}$ and magnetic pressure for each rotational phase of the two stars according to Eq. 2.1. The methodology of analysis are summarized as follows:

- Finding stellar parameters from photometric and spectroscopic data available.

- Determination of magnetic field geometry via analysis of available magnetic measurements.

- Verifying the effect of inhomogeneous surface distribution of chemical elements.

- Model atmosphere calculations with Lorentz force included for each rotational phase adjusting values of induced e.m.f. to match observed amplitude of standard deviation.

Table 1 lists the best fitted parameters used for modeling the Lorentz force effects in atmospheres of $\theta$ Aur and 56 Ari. Substantial errors in observations of the magnetic field variation found for 56 Ari did not allow at present to model magnetic field geometry other than dipole.

The best fit to the amplitude of the observed variations was obtained with $c_{1}=$ $1 \times 10^{-10}$ CGS (inward-directed force) and $c_{1}=1 \times 10^{-11}$ CGS (outward-directed force) for $\theta$ Aur and $c_{1}=5 \times 10^{-10}$ CGS (inward-directed force) and $c_{1}=7.5 \times 10^{-11}$ CGS (outward-directed force) in the case of 56 Ari.

\section{Acknowledgements}

This work was supported by FWF Lisa Meitner grant Nr. M998-N16 to DS.

\section{References}

Kroll, R. 1989, Rev. Mexicana AyA 2, 194

Shulyak, D., Valyavin, G., Kochukhov, O., et al. 2007, A\&A 464, 1089

Shulyak, D., Tsymbal, V., Ryabchikova, T., Stütz, Ch., \& Weiss, W. W. 2004, A $\& A 428,993$

Valyavin, G., Kochukhov, O., \& Piskunov, N. 2004, A\& $\&$ 420, 993 